\title{
Radiolabeled Immunoglobulin Therapy for Patients with Solid Tumors
}

\author{
Nabil Khater ${ }^{1,2 *}$, Marcel Kap ${ }^{3}$, Rima Sayah², Dimor Elbers ${ }^{4}$ and Huib M Vriesendorp ${ }^{1}$ \\ ${ }^{1}$ Phase 2 Therapy, The Hague, The Netherlands \\ ${ }^{2}$ Department of Radiation Oncology, Hotel-Dieu de France St. Joseph University Hospital, Beirut, Lebanon \\ ${ }^{3}$ Department of Pathology, Erasmus Medical Center, Rotterdam, The Netherlands \\ ${ }^{4}$ Lonza Ltd. Munchensteinerstrasse 38, CH-4002 Basel, Switzerland
}

\begin{abstract}
Purpose: To test the reactivity of monoclonal murine IgM and IgG for Tenascin-C (TNC) in formalinized solid human tumors tissue samples and to estimate the radiation dose that Yttrium- 90 labeled murine IgM reactive with TNC can deliver to a solid tumor and tumor containing draining lymph nodes.

Materials and Methods: Using Immunohistochemistry (IHC), mouse anti-human TNC IgM and IgG clones were tested for the detection of TNC in formalin fixed biopsies of patients with Glioblastoma multiforme (GBM), adeno carcinoma of the exocrine pancreas ( $\mathrm{PaCa}$ )-, breast-, colon-, renal-, ovary- prostate carcinoma, cutaneous-, ocular- melanomas, and Ewing Sarcoma. IHC was performed on all tumors with an $n=1$, except for $\mathrm{PaCa}$ and ocular melanomas, $n=11$. Monte-Carlo simulation and convolution calculations were used to determine the activity of $Y-90$ required for delivering $100 \mathrm{~Gy}$ to a $50 \times 50 \times 50 \mathrm{~mm}^{3}$ water-equivalent tumor model, assuming a homogeneous distribution of the radioimmunoconjugate throughout the model volume.
\end{abstract}

Results: IHC has confirmed reactivity of IgM with TNC in all of the tested human solid tumors samples except for ocular melanoma. Positive and negative controls of IgM specificity were used. The dosimetry simulation predicted an Yttrium-90 activity of $217 \mathrm{MBq}$ to deliver a dose of 100Gy to the tumor model with a $6 \mathrm{~mm}$ sharp dose fall off in surrounding normal tissues.

Conclusion: Loco-regional control of human solid tumors may be obtained with intra-tumoral administration of radiolabeled IgM targeting TNC. In TNC negative solid tumors such as ocular melanoma, other tumor-specific target(s) need to be identified.

Keywords: Radioimmunotherapy; Y-90; IgM; Intra-tumoral; Intralesional; Tenascin-C

\section{Introduction}

\section{Treatment of human patients with a solid tumor}

Approximately $50 \%$ of all solid tumor patients receive stateof-the-art local regional therapy with surgery and/or radiotherapy. Approximately half the patients with solid tumors receiving state of the art local therapy survive. They are cured. Approximately $25 \%$ of patients recur in the location of the primary tumor, the tumor draining lymph nodes and/or at distance by way of hematogenous metastases. Solid tumor patients with measurable hematogenous metastases before or after surgery/radiation are incurable but might obtain temporary benefit from systemic cancer treatment/chemotherapy [1,2].

\section{Retreatment of a solid tumor}

Repeat radiotherapy and/or surgery usually cannot be performed due to tolerance constraints of normal tissues surrounding the recurrence [2]. In those cases, local regional control may be achieved by intra-tumoral administration of a new form of Radiolabeled Immunoglobulin Therapy (RIT) targeting the Tenascin-C (TNC) component of the tumor, which is the subject of this report.

\section{TNC expression in normal and malignant tumors}

TNC expression is tightly regulated and generally repressed in healthy tissues. TNC is present in normal human breast and is reexpressed in damaged or infected tissues and in many human solid tumors. TNC occurs in different isoforms which are generated by alternative splicing and these TNC variants are abundantly expressed at the invasive tumor front [3]. TNC reactive IgM and an intra-tumoral route of administration are introduced to avoid off target effects [4-6].
Prior experience with radiolabeled IgG reactive with human TNC

Riva and Reardon $[7,8]$ treated patients with recurrent GBM by administering Iodine-131 labeled IgG reactive with human TNC, in the cyst created by the surgical resection of the recurrence. Survival of patients after I-131-IgG administration was often longer than the tumor response duration after initial treatment with surgical resection, post-op radiation and chemotherapy. The I-131 IgG radiation dose to the recurrence could not be determined accurately at the time and might have been too low for cures. Moreover, the gamma emissions of I-131 are too weak to deliver a homogeneous tumor dose and radioactive iodine is enzymatically removed from carrier molecules and taken up in the thyroid gland of the patient or secreted into the stomach of the patient $[4,5]$.

RIT studies in patients with Hodgkin's disease and nonHodgkin's Lymphoma

In the past, RIT has benefited patients with 'liquid' tumors, such as Hodgkin's disease (HD) and B-cell non-Hodgkin's lymphoma [5,9-13]. A low intravenous dose of $2 \mathrm{mg}$ of anti-ferritin rabbit IgG labeled with

*Corresponding author: Nabil Khater, Department of Radiation Oncology, HotelDieu de France St. Joseph University Hospital, Beirut, Lebanon, Tel: 9611604350 Fax: 9611604000; E-mail: nabil.khater@hdf.usj.edu.lb

Received June 23, 2017; Accepted August 01, 2017; Published August 07, 2017

Citation: Khater N, Kap M, Sayah R, Elbers D, Vriesendorp HM (2017) Radiolabeled Immunoglobulin Therapy for Patients with Solid Tumors. J Nucl Med Radiat Ther 8: 338. doi: 10.4172/2155-9619.1000338

Copyright: ( 2017 Khater N, et al. This is an open-access article distributed under the terms of the Creative Commons Attribution License, which permits unrestricted use, distribution, and reproduction in any medium, provided the original author and source are credited. 
Y-90 can have significant therapeutic effects [4,9-11]. In the absence of accurate dosimetric methods at the time, a positive dose effect curve could only be demonstrated in patients with HD by increasing the amount of Y-90 administered per kg bodyweight of the patient (not body surface area) from $111 \mathrm{MBq}$ to $148 \mathrm{MBq}$ and subsequently to $185 \mathrm{MBq}[9,11]$

Non-Hodgkin lymphoma patients treated with anti-CD-20 labeled with Y-90 need to receive a high $(200 \mathrm{mg})$ intravenous dose of monoclonal IgG to get tumor shrinkage [12]. Tumor responses are due to immunotherapy as well as radiotherapy, while responses in HD patients are easier to optimize because the tumor response is solely due to radiation [11].

\section{Prior translational research}

Translational research demonstrated that stable chelation of Y-90 to tumor-reactive IgM injected directly in a subcutaneous human tumor xenograft (diameter between $0.5-1.5 \mathrm{~cm}$ ) in nude mice diffused rapidly throughout the tumor. Ninety-five percent of the administered IgM was retained in the tumor, while approximately $5 \%$ reached the circulatory system. Human tumor xenografts in nude mice were sterilized in 1 or 2 treatment cycles for more than 200 days, without discernible clinical side-effects [14]. Histology and autoradiography showed Y-90 labeled IgM is soluble in vivo and diffuses through the tumor within three hours with the exception of necrotic areas in the tumor mass. Tumor shrinkage was observed $144 \mathrm{~h}$ after administration of Y-90 labeled IgM $[5,9,13,14]$. In contrast $50 \%$ of tumor-reactive IgG administered directly into the tumor was washed out in 2 days $[4,9,10,4,14]$. Aggregated albumen labeled with Y-90 did not diffuse through the whole tumor mass. It caused hot and cold spots within the tumor and caused less complete responses than tumor reactive $\mathrm{Y}-90$ labeled IgM $[7,9,14]$.

\section{Comparison of IgM and IgG}

Radiolabeled IgM and IgG behave differently in vivo due to differences in size and antigen binding sites (ABS). IgM is a pentamer with a molecular weight (MW) of $950 \mathrm{KDa}$ and $10 \mathrm{ABS}$, while IgG is a dimer with a $\mathrm{MW}$ of $165 \mathrm{KDa}$ and $2 \mathrm{ABS}$. IgM molecules cannot cross the blood-brain-barrier or the blood-tumor- barrier, therefore it can only exert a therapeutic effect, if it is administered directly into a tumor mass. The binding of $2 \mathrm{ABS}$ to TNC in the tumor is dynamic. When both $\mathrm{ABS}$ are no longer connected to TNC, tumor-reactive IgG is exported out of the tumor by way of tumor venules within 48 hours. Whereas, the $10 \mathrm{ABS}$ of IgM are rarely all disconnected from the tumor antigen and do not leave the tumor mass because IgM is too big to cross the tumor venule wall. IgM can and does emigrate from the tumor by way of large fenestrations in lymph vessel walls. IgM will stick to tumor contained in first echelon lymph nodes, while IgG does not [14-16].

These observations indicate that both, primary tumor and draining lymph nodes, can be treated with intra-tumoral administration of Y-90 labeled tumor reactive IgM. Y-90 is an exclusive beta emitter with 0.935 $\mathrm{MeV}$ average energy, maximum range of $10 \mathrm{~mm}$ in water, therapeutic range of near $5 \mathrm{~mm}$ and rapid fall-off thereafter.

\section{Materials and Methods}

\section{Immunohistochemistry}

All tissue samples used were taken from the paraffin block archives at the Pathology department of the Erasmus University Medical Center in Rotterdam, The Netherlands. In accordance with the Dutch Code of
Conduct for the use of residual tissue in research, the head of the tissue bank of the Erasmus University Rotterdam Medical Center graciously granted permission to use residual tissues for this research. The Human Protein Atlas [17] was consulted to find information on TNC expression in normal tissue. Positive control tissue sections (i.e. a multi tissue block containing; normal tonsil, intestine, liver and pancreas) of $4 \mu \mathrm{m}$ thickness were subjected to various methods of antigen retrieval (AR). The clones were tested without AR, with protease digestion ( $0.1 \%$ protease $\mathrm{K}$ for 5 minutes at room temperature) using DAKO, K1494, Dakocytomation (Denmark), and with heat induced epitope retrieval (HIER) i.e. cooking the sections in a temperature controlled microwave for 15 minutes at $100^{\circ} \mathrm{C}$ in a $\mathrm{pH} 9$ or $\mathrm{pH} 6$ Tris/EDTA buffer. Ten monoclonal IgGs and 10 monoclonal IgMs positive for human recombinant TNC in Western blots were provided by ProMab Biotechnologies Inc. (Richmond, CA, USA). All 20 anti-TNC clones; $10 \mathrm{IgG}$ and $10 \mathrm{IgM}$ were diluted 1:10, 1:100 and 1:1000 to determine the optimal dilution. As negative control, the primary antibody was omitted. Slides were incubated with the primary antibodies for 30 minutes at room temperature and with diaminobenzidine for 10 minutes at room temperature using DAKO REAL EnVison, K5007 (Denmark) to detect the tissue bound primary antibody. The sections were counterstained with hematoxylin for 1 minute and cover slipped. The optimal TNC staining, hence the optimal protocol, was selected by comparing the TNC staining results to the images in the Protein Atlas data base of normal tissues [17].

A tissue micro array (TMA) consisting of pancreas carcinoma samples of 11 different patients was made. A pathologist marked the tumor sites on the hematoxylin and eosin stain slide of the corresponding paraffin block. By use of a Beecher manual tissue arrayer, $1 \mathrm{~mm}$ cores were taken from the selected tumor blocks (1 core from each block) and gathered in a receptor block TMA. Sections of $4 \mu \mathrm{m}$ thickness were cut and immunohistochemistry (IHC) was performed using the optimized protocol. On one slide 11 pancreas carcinoma samples were stained, all under the same conditions to ensure differences in TNC expression (staining intensity) are not related to inter-assay variability. Digital images of all relevant slides were exported from scanned slides (Hamamatsu Nanozoomer, Hamamatsu, Germany).

\section{Dosimetry}

A convolution of the bio-distribution with the dose point kernel (DPK) will result in a 3D dose distribution [18]. For computational purposes a kernel with finite voxel size is required and termed herein dose voxel kernel (DVK). Using the MCNPX Monte Carlo code version 2.6 c [19], the DVK of Y -90 in water was simulated in Cartesian cubical geometry at $0.5 \mathrm{~mm}, 1 \mathrm{~mm}$ and $3 \mathrm{~mm}$ voxel sizes, and the DPK was simulated in concentric shells geometry for a near-point- source of 20 $\mu \mathrm{m}$ radius with $20 \mu \mathrm{m}$ shell thicknesses. The DVK's at $0.5 \mathrm{~mm}$ and 1 $\mathrm{mm}$ voxel sizes were used for convolution computations, and the DVK at $3 \mathrm{~mm}$ voxel size was used to validate the results by comparison with MIRD-17 S values [20] also calculated at a voxel size of $3 \mathrm{~mm}$. The DPK was used to calculate the radius of the sphere $\left(\mathrm{X}_{90}\right)$ within which $90 \%$ of the dose is absorbed. For the $1 \mathrm{~mm}$ voxel DVK, the source size was modeled at $1 \times 1 \times 1 \mathrm{~mm}^{3}$ located at the center of cubic voxel geometry. Source strength was modeled homogenously distributed inside the source. Tallies were averaged in each voxel, and values were approximated to represent dose at the center of each voxel, in a volume spanning $25 \times 25 \times 25 \mathrm{~mm}^{3}$. In concentric shells geometry, the point source was modeled as a spherical source with $20 \mu \mathrm{m}$ radius. Energy was tallied in concentric shells around the source with shell thicknesses constant at $20 \mu \mathrm{m}$. The outer shell radius was set to $12 \mathrm{~mm}$; the shell 
tally was approximated to represent dose at mid-distance from outer and inner shell boundaries. The medium in both geometries was set to water. The spectrum of Y-90 was extracted from Cross et al. [21] and modeled with equally spaced energy bins of $0.25 \mathrm{MeV}$. The electron and photon cutoff energy set to $1 \mathrm{keV}$. Photons and electrons were tracked and F6 tallies (electron+photon) were used to calculate the dose in each voxel. Ten million histories were followed. The 3D DVK was regenerated using a MATLAB script (The MathWorks, Natick, MA) in a volume of $25 \times 25 \times 25 \mathrm{~mm}^{3}$ with a $1 \mathrm{~mm}$ voxel size. A hypothetical planning target volume (PTV) was also modeled in the script as a 50 $\times 50 \times 50 \mathrm{~mm}^{3}$ of water equivalent material with a $1 \mathrm{~mm}$ voxel size, a uniform biodistribution of 37Bq/voxel ( $1 \mathrm{nCi} /$ voxel), and surrounded by water. Convolution of the PTV with the 3D DVK was performed using MATLAB to estimate the activity of Y-90 needed to deliver a dose of $100 \mathrm{~Gy}$ to the PTV. The matrix dimension resulting from the full convolution of the PTV with the DVK is $74 \times 74 \times 74 \mathrm{~mm}^{3}$, permitting the recording of dose in the gradient just outside the PTV in addition to the dose within the PTV. The same calculations were repeated for 0.5 $\mathrm{mm}$ voxel kernel and $0.5 \mathrm{~mm}$ voxel PTV to assess voxel size effect on dosimetry. The convolution will yield an initial dose rate (D) decaying exponentially with the half-life (T1/2) (64 h for Y-90).

The integral dose inside the PTV (D்) was calculated as follows:

$$
D s=\dot{\mathrm{D}}(1.44) \mathrm{T}_{1 / 2}
$$

Where: the PTV [22].

The total activity inside the PTV used in this simulation As was calculated as follows:

As $=V \cdot a$

Where:

- $V$ is the PTV volume in voxels

- $a$ is the activity per voxel (37 Bq)

The total activity $A_{T}$ required to deliver an integral total dose $D_{T}$ of $100 \mathrm{~Gy}$ to the PTV calculated as follows:

$$
A_{T}=A_{S} \cdot D_{T} / D_{S}
$$

\section{Results}

\section{IHC studies in formalin fixed tissues of various human tumors}

Eight out of 10 monoclonal IgG's and 6 out of 10 IgM's recognized TNC in human tissues by IHC. One of the 6 monoclonal IgM's that reacted with TNC in human tissues by IHC was selected for making a master cell bank. The expression patterns found in normal tonsil, intestine, liver and pancreas tissue resembled the patterns found in the human protein atlas [17] confirming the specificity for TNC for both IgG and IgM.

\section{TNC expression in a GBM resection specimen}

Mouse monoclonal IgG and IgM reacted with TNC in the extracellular matrix surrounding the tumor cells and in some individual cells (brown staining in Figure 1). The monoclonal IgM anti- TNC has a 10-fold lower titer than the monoclonal IgG. Mouse spleen cells were harvested after 6 weekly immunizations. IgG will increase in affinity and titer after each immunization. In contrast IgM titers decrease more rapidly overtime, and repeat immunizations will each time produce the same spike of antigen reactive IgM, with the same low affinity [23] (Figure 1).

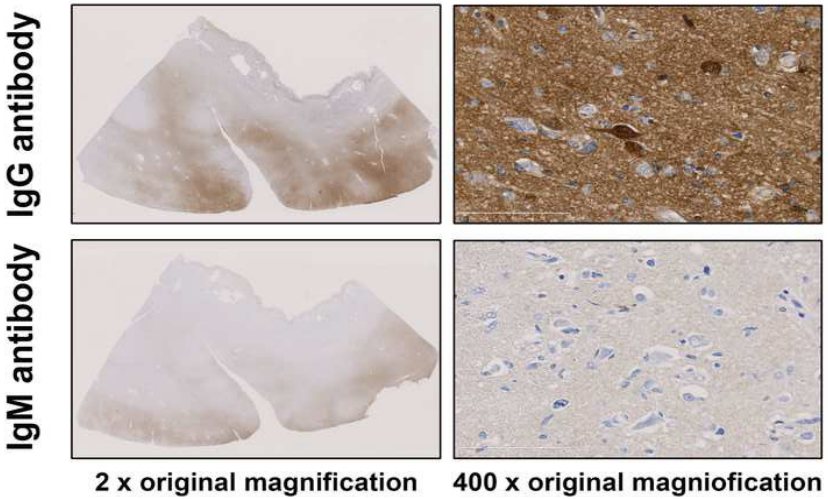

Figure 1: TNC expression in GBM. Dark staining areas contain GBM cells Optimal lgG titer is $1: 100$. Optimal IgM titer is $1: 10$.

\section{TNC Expression in Adenocarcinoma of the Exocrine Pancreas (PaCa)}

IgG and IgM reacted with TNC in $11 / 11$ patients with $\mathrm{PaCa}$. TNC expression is quite heterogeneous as observed in 4 patient samples shown in Figure 2.

\section{TNC expression in other human solid tumors}

IgG and IgM reacted with TNC in one sample of colon-, renal(Grawitz tumor), prostate-, breast- carcinoma, Ewing sarcoma, ovarian cancer and melanoma. Eleven different patient samples of ocular melanoma were TNC-negative. In most other tumors tested TNC is detected in the connective tissue surrounding the tumor cells. In the breast carcinoma sample the tumor cells themselves contain TNC (Figure 3).

\section{Y-90 Dose Point and DVK}

Good agreement is observed between the four simulation geometries presented and MIRD-17 (Figure 4). The $3 \mathrm{~mm}$ DVK is almost identical to the MIRD-17 S values also calculated at $3 \mathrm{~mm}$ voxel size. Due to voxel volume averaging, the dose rate increases with decreasing voxel size at distances less than $2 \mathrm{~mm}$ from the source center and decreases with increasing voxel size at distances greater than $6 \mathrm{~mm}$ from the source. The DPK exhibits a high dose rate in proximity of a $20 \mu \mathrm{m}$ point source due to the singularity problem. Beyond $10 \mathrm{~mm}$, the dose contribution is nil. $\mathrm{X}_{90}$ was evaluated from the concentric shells simulation to be $0.533 \mathrm{~cm}$, which compares well with $5.34 \mathrm{~mm}$ reported by Simpkin [24] and $5.4 \mathrm{~mm}$ reported by Botta [25]. Consequently, this comparison appears to justify the use of simulated kernels in the dose convolution calculations (Figures 4 and 5).

\section{Dosimetry estimation}

The convolution of the $1 \mathrm{~mm}$ voxel DVK with the PTV at the assumed biodistribution of $37 \mathrm{~Bq} /$ voxel (1nCi/voxel), yielded an initial dose rate (D) of $23.2 \mathrm{mGy} / \mathrm{hr}$. $\dot{\mathrm{D}}$ is homogeneous inside the PTV with a sharp falloff to 0 at $6 \mathrm{~mm}$ outside the PTV (Figure 5).

The integral dose inside the PTV (Ds) was $2.13 \mathrm{~Gy}$ calculated using equation (1).

The total activity inside the PTV used in this simulation $(A s)$ was 4.625 MBq calculated using equation (2). 

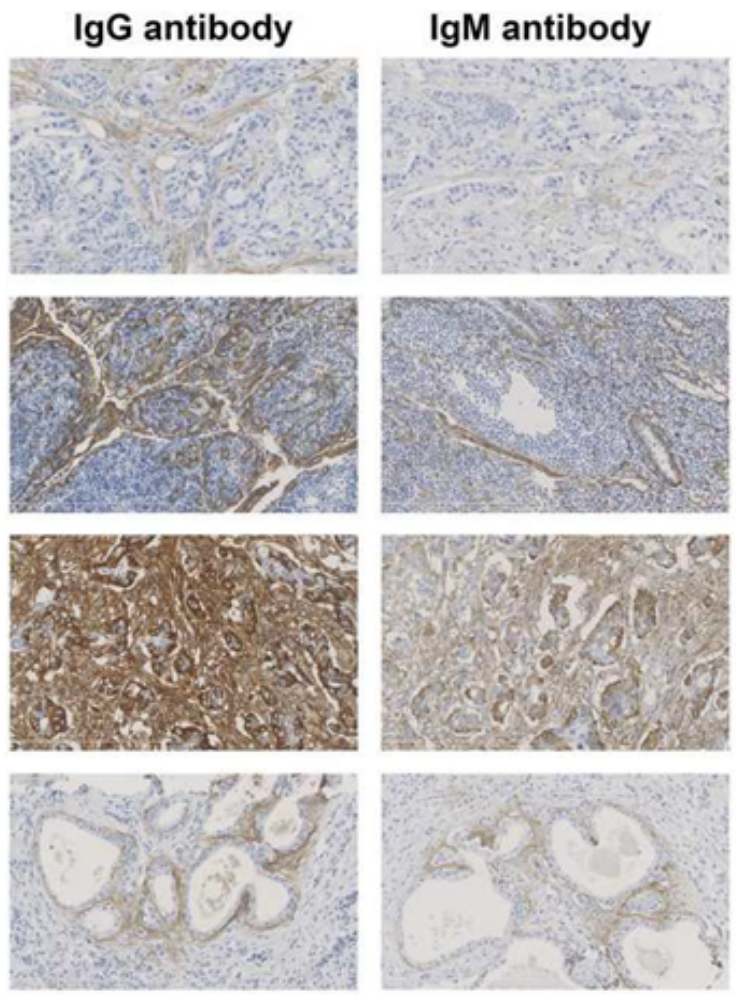

$100 x$ original magnification

Figure 2: TNC expression in 4 human pancreatic tumors. Dark areas indicate the presence of TNC.

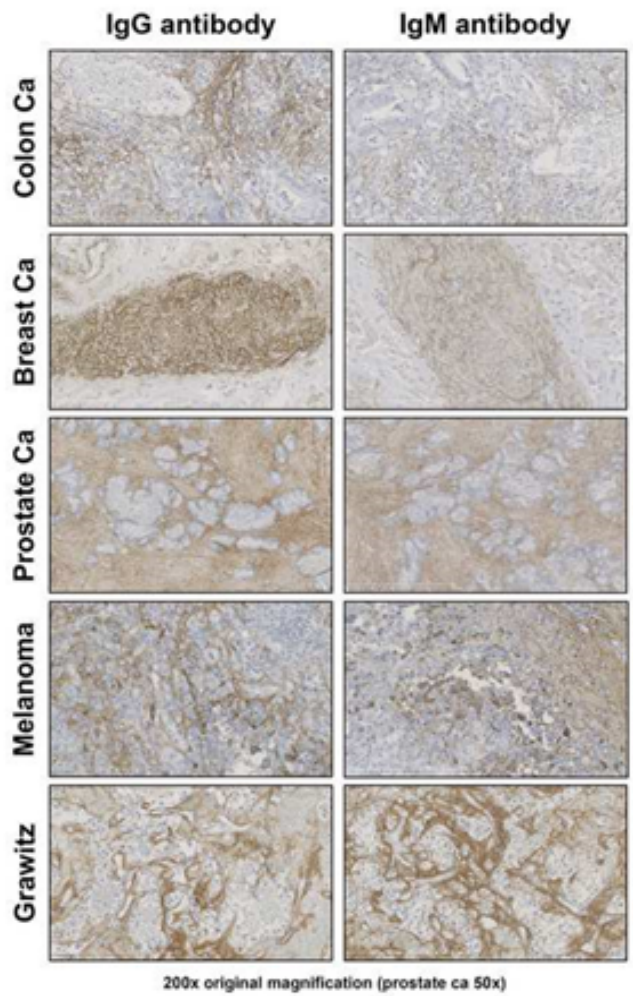

Figure 3: TNC expression in several human solid tumors. Dark areas indicate the presence of TNC.

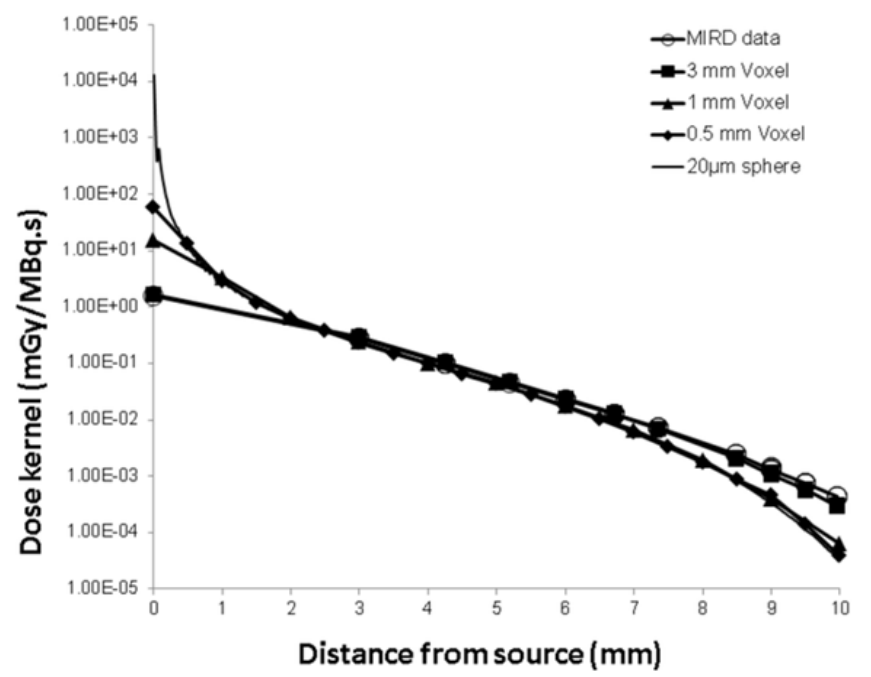

Figure 4: Y90 DVK's of $0.5 \mathrm{~mm}, 1 \mathrm{~mm}$ and $3 \mathrm{~mm}$ voxel sizes and DPK for a 20 $\mu \mathrm{m}$ point source compared to MIRD-17 [20].

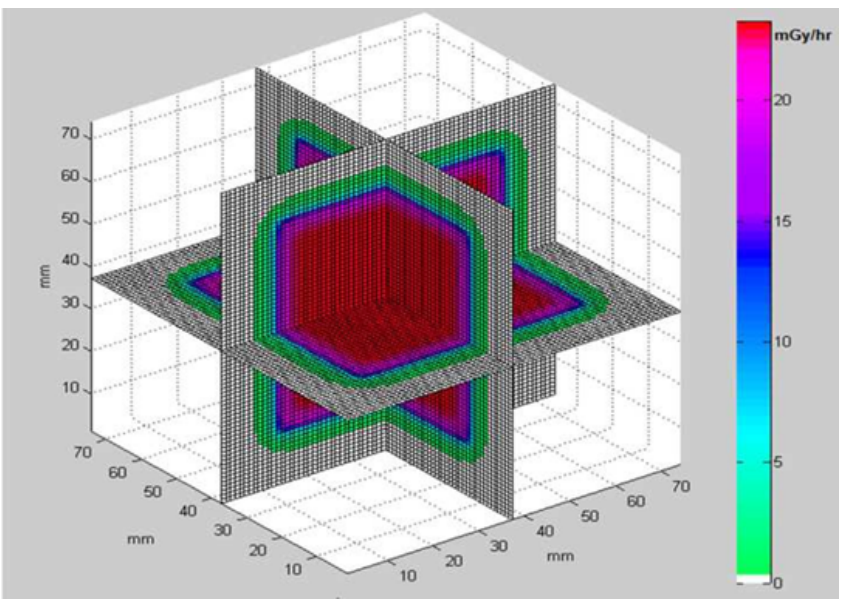

Figure 5: Dose rate $(\mathrm{mGy} / \mathrm{hr})$ distribution within the PTV model with $1 \mathrm{~mm}$ voxel size containing 37Bq/voxel. The computed dose rate at the core is $23.2 \mathrm{mGy} /$ $\mathrm{hr}$ (red area); then tapers to $0 \mathrm{mGy} / \mathrm{h}$ (outer edge of green area) over a distance of $6 \mathrm{~mm}$.

The total activity $\left(A_{T}\right)$ required to deliver an integral total dose $\left(D_{T}\right)$ of 100Gy to the PTV was $217 \mathrm{MBq}$ calculated using equation (3).

Calculations were repeated for the same PTV model and DVK but with $0.5 \mathrm{~mm}$ voxel size, yielding $A_{T}$ value of $226 \mathrm{MBq}$ (Table 1); not significantly different from the $1 \mathrm{~mm}$ voxel size calculations. In this simulation, it is assumed that no activity is metabolized biologically. In vivo, immune complexes of antigen and immunoglobulin are catabolized slower than the half-life of the bare, not antigen binding, immunoglobulin [5] (See "Pharmaco-kinetics and bio-distribution" and "Pharmaco-vigilance and patient safety" sections for effective halflife and residence time determination).

\section{Discussion}

Results reported in this communication, prior translational animal studies, and RIT studies in patients with end-stage Hodgkin's disease [4-6,9,26], suggest that a high 'targeted' dose with sharp dose fall-off in 


\begin{tabular}{|c|c|c|c|c|}
\hline $\begin{array}{c}\text { Voxel size } \\
(\mathbf{m m})^{3}\end{array}$ & $\mathbf{V}$ (voxels) & $\dot{\mathbf{D}}^{(1)}(\mathbf{m G y} / \mathbf{h r})$ & $\mathbf{D}_{\mathbf{s}}^{(2)}(\mathbf{G y})$ & $\mathbf{A}_{\mathbf{T}}^{(3)}(\mathbf{M B q})$ \\
\hline$(1 \mathrm{~mm})^{3}$ & $1,25,000$ & 23.2 & 2.13 & 217 \\
\hline$(0.5 \mathrm{~mm})^{3}$ & $10,00,000$ & 177 & 16.3 & 226 \\
\hline
\end{tabular}

D is the simulated initial dose rate. ${ }^{(1)}$

$D_{\mathrm{S}}$ is the integral simulated dose resulting from $37 \mathrm{~Bq} /$ voxel. ${ }^{(2)}$

$A_{\mathrm{T}}$ is the simulated therapeutic activity that would deliver $100 \mathrm{~Gy}$ to the PTV. ${ }^{(3)}$

Table 1: PTV model simulated dosimetry for 2 different voxel sizes.

adjacent normal tissues may be delivered to patient with solid tumors via intra-tumoral RIT using Y-90 labeled murine IgM against TNC. TNC appears to be a good target as it is abundant in most solid tumors tested and has limited expression in healthy normal tissues.

\section{Radiation biology}

The dose rate after RIT decreases exponentially with the half-life of the radionuclide. In general, biological effects of radiation; such as cell killing, tend to decrease with lower dose rates [27,28]. However, cells irradiated at low dose rates tend to collect in the G2M phase of the cell cycle, in which, the DNA condenses, and lethal double strand DNA breaks become more frequent; double DNA strand breaks are the dominant killing mechanism for tumors as well as normal cells. As a result, Gray for Gray low dose rate RIT and daily fractions of external beam radiation therapy (EBRT) doses are almost equally effective. Theoretically several RIT cycles could be sufficient to obtaining local regional control of a solid tumor without serious side effects. This makes this therapy much more effective than EBRT in terms of escalating the dose while maintaining normal tissue tolerance. Hence new possibilities will be opened for treating tumors known to be nonresponsive to EBRT.

\section{Pharmaco-kinetics and bio-distribution}

In prior experiments with human tumor xenograft bearing nude mice the administration of In-111 and Y-90 labeled immunoglobulins showed similar pharmacokinetics and biodistributions. This justifies the use of the 3D In-111 distribution as an accurate predictor for the in vivo Y-90 distribution $[4,5,9,11,13,26]$. In-111 is a gamma-emitter with $67 \mathrm{~h}$ half-life; close to that of Y-90 (64 h). It has two energy peaks that can be accurately detected using properly collimated gamma camera. Serial SPECT imaging on 3,24,48 and 72 hours after administration will verify the residence time in vivo. It also allows the determination of the bio-distribution and the effective half-life, hence permitting patientspecific dosimetry, where the SPECT measured effective half-life would be used for dose computation in equation (1). Y-90 linked and In-111 linked proteins can only be expected to provide similar biodistributions if a stable linker with 8 co- ordination points is used, such as $2 \mathrm{~B}, 3 \mathrm{M}$, DTPA [29]. Other clinically used chelates without carbon backbone stabilization have only 7 co-ordination points, thereby chelated In-111 which only needs 6 co-ordination points would be bound in a stable manner, but Y-90, a bone seeker, needs 8 co-ordination points. With chelates having only 7 co-ordinates points, Y-90 would be released in vivo, which will cause serious bone marrow damage in human cancer patients.

\section{Pharmaco-vigilance and patient safety}

To ascertain that only patients who might benefit from this therapy will be selected for treatment, radiolabeled IgM reactive with human TNC should be delivered in two steps $[4,5,9,11,13]$ :

Step 1: Intra-tumoral administration of $2 \mathrm{mg}$ of a mouse monoclonal IgM labeled with Indium-111 (In-111) reactive with TNC.
This provides two important advantages:

1. In vivo determination of the location, stability and residence time of the radio-immuno-conjugate. Patients with insufficient radio-immuno-conjugate in the tumor or too much radio-immunoconjugate in critical normal tissues will not benefit from step 2 RIT and will not proceed to step 2 .

2. Treatment planning and patient-specific dosimetry to determine the activity required and resulting dose distribution. The In-111 biodistribution acting as a predictor of the Y-90 biodistribution will be used in the dose calculation. The dosimetry would be specific to the tumor and normal tissue volumes of the individual patient. More accurate normal tissue and solid tumor tissue dose response curves will become available. The $1 \mathrm{D}$ and $2 \mathrm{D}$ measures for tumor staging and tumor response can be replaced by 3D (cc, tumor volume measures).

Step 2: One week after successful In-111 labeled IgM administration, $2 \mathrm{mg}$ of Y-90 labeled IgM reactive with TNC will be administered directly into the tumor.

\section{Clinical radio-pharmacy}

The radioimmunoconjugate (RIC) administered to human patients should always be protected from radiolysis by adding endotoxin-free clinical grade Human Serum Albumin. Once radiolabeled, the IgM should be administered within $4 \mathrm{~h}$. Radiolysis of the In-111 or Y-90 labeled RIC has not been observed in the radio-pharmacy by instant or thin layer chromatography. This was demonstrated in more than 200 RIT cycles in 90 patients with 4-5 sequential blood samples/patient taken in the first week after administration $[5,11,13]$. In vivo radiolysis of the In-111 labeled IgG has occurred in patients with circulating antibodies against murine IgG. In-111 is not a bone-seeker like Y-90, but secreted by way of bile into the digestive tract [11].

\section{Optimization of the radioimmunoconjugate}

The replacement of low TNC affinity genes in the variable region of the IgM with high affinity variable genes of anti TNC IgG will allow for a more economical smaller mg protein dose per RIT administration [23]. Humanization of the murine IgM will reduce anti-antibody formation and lower the risks of using this IgM in human patients more than once [30]. The murine IgM will be assessed for post-translational motifs (PTMs) which could increase the risks in recombinant manufacturing and decrease its shelf life. Removal of risky PTMs can reduce aggregation and increase stability and shelf life of the IgM-DTPA [31-33].

\section{Proposed human studies}

Intra-tumoral administration of Y-90 labeled IgM reactive with TNC should be explored in patients with poor prognosis solid tumors such as recurring GBM and PaCa. Almost all GBM patients succumb to their disease within 4 years after the initial diagnosis or within 2 years after a recurrence. Most GBMs recur close to the location of the original mass. Dose escalation studies in patients with a recurrent GBM will have to start at a low dose because all of them will have received high EBRT dose previously. Half of the patients with a resectable $\mathrm{PaCa}$ are cured by radical surgery with a surgical mortality rate of 2-4\% [34]. The overall long term survival rate of all $\mathrm{PaCa}$ patients is $<10 \%$. Risk factors for recurrent $\mathrm{PaCa}$ patients after radical surgery are regional lymph nodes positive for $\mathrm{PaCa}$ and/or close or positive margins in the surgical resection specimen. In this report formalinized tumor biopsies of 11 out of 11 patients with $\mathrm{PaCa}$ tested positive for TNC in their tumor matrix. Pre-op use of intra-tumoral IgM reactive with TNC in patients with borderline operable $\mathrm{PaCa}$ might increase local regional 
Citation: Khater N, Kap M, Sayah R, Elbers D, Vriesendorp HM (2017) Radiolabeled Immunoglobulin Therapy for Patients with Solid Tumors. J Nucl Med Radiat Ther 8: 338. doi: 10.4172/2155-9619.1000338

Page 6 of 6

control of $\mathrm{PaCa}$ and increase the applicability of the only curative option for $\mathrm{PaCa}$ at this time; radical surgery [34]. The inhomogeneous distribution of TNC in the $\mathrm{PaCa}$ samples tested in this study will not lead to cold spots in the tumor volume due to the relatively long therapeutic range $\left(\mathrm{X}_{90}=5.33 \mathrm{~mm}\right)$ of $\mathrm{Y}-90$. If proof of principle can be obtained in RIT studies of GBM and PaCa patients, other solid tumor patients with TNC in their tumor matrix can be studied and a search for other tumor matrix antigens in TNC negative tumor, such as ocular melanoma, should be made.

\section{Conclusion}

The IHC results provided proof that monoclonal IgM and IgG can recognize TNC in several human tumor tissue samples including GBM and PaCa. IgG has higher affinity for TNC than IgM. The tumor model dosimetry simulation estimated the Y-90 activity required in a therapeutic RIT cycle to deliver the tumor targeted high dose while sparing normal tissues. This study in combination with prior translational animal research indicate that loco-regional control of human solid tumors may be obtained with intra-tumoral administration of Y90-labeled tumor reactive IgM. The exploration of this therapy in short single arm Phase 2 studies in patients with thus far non-curable tumors such as GBM and $\mathrm{PaCa}$, will provide information on how to optimize survival or even cure.

\section{Acknowledgments}

Developing new applications for Radiolabeled Immunoglobulin Therapy requires a multidisciplinary team. Huib Vriesendorp wants to acknowledge his long standing collaboration with Syed M Quadri, $\mathrm{PhD}$, an excellent friend, educator and chemist and Paul Borchard, PhD, for their valuable contributions to the field of radioimmunotherapy. This research did not receive any specific grant from funding agencies in the public, commercial, or not-for-profit sectors.

\section{References}

1. DeVita VT, Lawrence S, Rosenberg SA, Da Pinho RA, Weinberg RA (2014) De Vita, Hellman, and Rosenberg's Cancer Principles \& Practice of Oncology. (10 edtn), Wolters Kluwer, USA

2. Halperin EC, Brady LW, Perez CA, Wezer DE (2013) Perez and brady principles and practice of radiation oncology. 6th edition, Wolters Kluwer, USA.

3. Lowy CM, OskarssonT (2015) Tenascin C in metastasis: A view from the invasive front. Cell Adh Migr 9: 112-124.

4. Vriesendorp HM, Quadri SM, Borchardt PE (1998) Tumor therapy with radiolabeled antibodies, optimisation of therapy. BioDrugs 10: 275-293.

5. Vriesendorp HM, Quadri SM (2001) Radiolabeled immunoglobulin therapy: Old barriers and new opportunities. Expert Rev Anticancer Ther 1: 461-478.

6. Borchardt PE (1997) Radiolabeled human monoclonal IgM for intracompartmental cancer therapy.

7. Riva P, Arista A, Tison V, Sturiale C, Franceschi G, et al. (1994) Intralesional radioimmunotherapy of malignant gliomas: An effective treatment in recurrent tumors. Cancer 73: 1076-1082.

8. Reardon DA, Akabani G, Coleman RE, Friedman AH, Friedman HS, et al (2002) Phase II trial of murine (131) I-labeled antitenascin monoclonal antibody 81C6 administered into surgically created resection cavities of patients with newly diagnosedmalignant gliomas. J Clin Oncol 20: 1389-1397.

9. Vriesendorp HM, Quadri SM (2000) Radiolabeled immunoglobulin therapy in patients with Hodgkin's disease. Cancer Biother Radiopharm 15: 431-445.

10. Vriesendorp HM, Vriesendorp FJ (2003) A review of the intravenous administration ofradiolabeled Immunoglobulin $\mathrm{G}$ to cancer patients: High or low protein dose? Cancer Biother Radiopharm 18: 35-46.

11. Vriesendorp HM, Morton JD, Quadri SM (1995) Review of five consecutive studies of radiolabeled immunoglobulin therapy in Hodgkin's disease. Cancer Res 55: 5888s-5892s.

12. Knox SJ (1995) Radioimmunotherapy of the non-Hodgkin's lymphomas. Semin Radiat Oncol 5: 331-341.
13. Lai J, Quadri SM, Borchardt PE, Harris L, Wucher R, et al. (1999) Pharmacokinetics of radiolabeled polyclonal antiferritin in patients with Hodgkin's disease. Clin Canc Res 5: 3315s-3323s.

14. Borchardt PE, Quadri SM, Freedman RS, Vriesendorp HM (1997) Intralesional radiolabeled human monoclonal IgM in human tumor xenografts. Radiother Oncol 44: 283-293.

15. Borchardt PE, Quadri SM, Freedman RS, Vriesendorp HM (2000) Intraperitoneal radioimmunotherapy with human monoclonal IgM in nude mice with peritoneal carcinomatosis. Cancer Biother Radiopharm 15. 53-64.

16. De Jager R, Abdel-Nabi H, Serafini A, Pecking A, Klein JL, et al. (1993) Current status of cancer immunodetection with radiolabeled human monoclonal antibodies. Semin Nucl Med 23: 165-179.

17. The Human Protein Atlas.

18. Giap HB, Macey DJ, Bayouth JE, Boyer A (1995) Validation of a dose-poin kernel convolution technique for internal dosimetry. Phys Med Biol 40: 365-381.

19. Hendricks JS, McKinney GW, Durkee JW, Finch ML, Fensin MR, et al (2006) MCNPX, Version 26C. Report: LA-UR-06-7991, Los Alamos National Laboratory, USA.

20. Bolch WE, Bouchet LG, Robertson JS, Wessels BW, Siegel JA, et al. (1999) MIRD pamphlet No. 17: The dosimetry of nonuniform activity distributionsradionuclide $S$ values at the voxel level. Medical Internal Radiation Dose Committee. J Nucl Med 40: 11S-36S.

21. Cross WG, Ing H, Freedman N (1983) A short atlas of beta-ray spectra. Phys Med Biol 28: 1251-1260.

22. Khan FM, Gibbons JP (2014) Khan's the physics of radiation therapy. (5 $5^{\text {th }}$ Edtn), Lippincott Willams \& Wilkins, USA.

23. Hood LE, Weisman IL, Wood WB, Wilson JH (1994) Immunology. (2 ${ }^{\text {nd }}$ edtn), Benjamin/Cummings Publishing Co, USA

24. Simpkin DJ, Makie TR (1994) EGS4 Monte Carlo determination of the beta dose kernel in water. Med Phys 17: 179-186.

25. Botta F, Mairani A, Battistoni G, Cremonesi M, Fasso A, et al. (2011) Calculation of electron and isotopes dose point kernels with FLUKA Monte Carlo code for dosimetry in nuclear medicine therapy. Med Phys 38: 3944-3954

26. Vriesendorp HM, Quadri SM, Anderson BS, Wyllie CT, Dicke KA (1997) Recurrence of Hodgkin's disease after In-111 andYttrium-90 labeled antiferritin administration. Cancer 80: 2721-2727.

27. Hall EJ (2000) Radiobiology for the radiologist. (5th edtn), Lippincott Williams \& Williams, Philadelphia, USA.

28. Dillehay LE, Williams JR (1990) The present and future role of monoclona antibodies in the management of cancer. Front Radiat Ther Oncol 24: 96-103.

29. Quadri SM, Mohammedpour H (1992) Synthesis of 2-p- Aminobenzyl-3Methyland 2-p- Benzylderivatives of Diethylene Triamine Penta Aceticacid (DTPA): Carbon backbone modified bifunctional chelating agents. Bioorg Med Chem Lett 2: 1661-1664.

30. Van Walle I, Gansemans Y, Parren PW, Stas P, Lasters I (2007) Immunogenicity screening in protein drug development. Expert Opin Biol Ther. 7: 405-418.

31. Zurdo J, Arnell A Obrezanova O, Smith N, de la Cuesta RG et al. (2015) Early implementation of $\mathrm{QbD}$ in biopharmaceutical development: A practical example.

32. Obrezanova O, Arnell A, de la Cuesta RG, Berthelot ME, Gallagher TR, et al. (2015) Aggregation risk prediction for antibodies and its application to biotherapeutic development. MAbs 7: 352-363.

33. Zurdo J (2013) Developability assessment as an early de-risking tool for biopharmaceutical development. Pharm Bioprocess 1: 29-50.

34. Ryan DR, Manon H (2013) Management of locally and borderline resectable exocrine pancreatic cancer. 